\title{
Acknowledgement to Reviewers of Antibodies in 2019
}

\author{
Antibodies Editorial Office \\ MDPI, St. Alban-Anlage 66, 4052 Basel, Switzerland \\ Published: 30 January 2020
}

The editorial team greatly appreciates the reviewers who have dedicated their considerable time and expertise to the journal's rigorous editorial process over the past 12 months, regardless of whether the papers are finally published or not. In 2019, a total of 54 papers were published in the journal, with a median time to first decision of 16 days and a median time from submission to publication of 43 days. The editors would like to express their sincere gratitude to the following reviewers for their generous contribution in 2019:

\author{
Alam, Md Kausar \\ Al-Saleem, Fetweh \\ Álvarez-Vallina, Luis \\ Ban, Bhupal \\ Barb, Adam W. \\ Barroso, Margarida \\ Bawadekar, Mandar \\ Benhar, Itai \\ Billiald, Philippe \\ Bowen, Anthony \\ Brinson, Robert \\ Burvenich, Ingrid \\ Caaveiro, Jose \\ Challa, Dilip \\ Chan, Kuan Rong \\ Cheng, Hao \\ Conroy, Paul \\ Cretich, Marina \\ De Kruif, John \\ De Taeye, Steven W. \\ Delputte, Peter \\ Devoogdt, Nick \\ Dixit, Surjit \\ Drake, Penelope M. \\ Dyson, Michael \\ Eboigbodin, Kevin \\ Escobar-Cabrera, Eric \\ Fischer, Nicolas \\ Ghag, Gaurav \\ Ghiggeri, Gian Marco \\ Gisterå, Anton \\ Gorovits, Boris \\ Grönwall, Caroline
}

\author{
Healey, Gareth \\ Henry, Kevin \\ House, Imran \\ Hsieh, Ming-Ching \\ Imamura, Hiroshi \\ Ippolito, Gregory \\ Jamin, Christophe \\ Jones, Martina \\ Kim, Kristine M. \\ Kontermann, Roland \\ Koromyslova, Anna \\ Krause, Frank \\ Leusen, Jeanette \\ Li, Jinping \\ Liu, Bin \\ Liu, Xianglei \\ Lucas, Andrew T. \\ Lyu, Yuanzhi \\ Ma, Buyong \\ Malik, Ravinder \\ Malmström, Johan \\ Matikonda, Siddharth \\ Menzel, Stephan \\ Meulenberg, Eline \\ Mølhøj, Michael \\ Mueller, Philipp \\ Mukherjee, Somnath \\ Muyldermans, Serge \\ Nesterov-Mueller, Alexander \\ Nijhof, Inger \\ Novak, Jan \\ Opaliński, Łukasz \\ Ozen, Mehmet
}


Pegu, Amarendra

Perkins, Stephen J.

Platts-Mills, Thomas A.

Ponraj, Prabakaran

Porollo, Aleksey

Rasche, Leo

Ren, Gang

Rissiek, Bjoern

Ronsard, Larance

Rossotti, Martin A.

Sahni, Sanjeev K.

Samoylova, Tatiana I.

Sautto, Giuseppe A.

Schmidt, Florian

Seifert, Oliver
Shim, Hyunbo

Strohl, William R.

Sun, Wei

Sundaram, Kruthika

Tabares, Sofia

Torres, Raul

Tscheliessing, Rupert

Van Leeuwen, Fijs W.B.

Vance, David

Vattepu, Ravi

Wang, Lai-Xi

Weiss, Siegfried

Wilson, Patrick

Wozniak-Knopp, Gordana

Yasunaga, Masahiro

(C) 2020 by the author. Licensee MDPI, Basel, Switzerland. This article is an open access article distributed under the terms and conditions of the Creative Commons Attribution (CC BY) license (http://creativecommons.org/licenses/by/4.0/). 